Turk. J. Math. Comput. Sci.

13(2)(2021) 318-330

(C) MatDer

DOI : $10.47000 /$ tjmcs. 909498

\title{
Hermite-Hadamard Type Inequalities Related to s-Convex Functions in the Third Sense
}

\author{
ZEYNEP EKEN ${ }^{10}$ \\ Faculty of Education, Akdeniz University, 07058, Antalya, Turkey.
}

Received: 04-04-2021 • Accepted: 09-08-2021

Aвstract. In this paper, some Hermite-Hadamard type inequalites for $s$-convex functions in the third sense are studied. It is established several new inequalities for functions whose derivative in absolute value and $p$ th power of its derivative in absolute value are $s$-convex in the third sense. In addition, these inequalities are used to find an upper bound for error in numerical integration for this type of functions.

2010 AMS Classification: 26A51, 26D15

Keywords: Hermite-Hadamard inequality, s-convex function in the third sense.

\section{INTRODUCTION}

Convex functions play an important role in many branches of mathematics, accordingly in other areas of science as well. In parallel with the developments in the fields of science, many different generalizations of the concept of convexity have been introduced [1-3,6-9,16,18,19,23]. $s$-Convexity in the third sense studied in [14] is one of them.

On the other hand, one of the most important properties of convex functions is that they satisfy certain inequalities such as Jensen and Hermite-Hadamard inequalities. The Hermite-Hadamard inequality is known in the literature as

$$
f\left(\frac{a+b}{2}\right) \leq \frac{1}{b-a} \int_{a}^{b} f(x) d x \leq \frac{f(a)+f(b)}{2}
$$

where $f:[a, b] \rightarrow \mathbb{R}$ is a convex function on the interval $[a, b]$.

In literature, the Hermite-Hadamard inequality for the generalizations of the convex functions is obtained by many researchers [4,10-12,24]. As a continuation of these works, the extensions and refinements of the Hermite-Hadamard inequality for these functions satisfying certain condations have been the subject of many studies [5, 13, 15, 17, 20-22, 25].

In this study, we will focus on Hermite-Hadamard type inequalities for the functions whose absolute value of its derivative is $s$-convex functions in the third sense and obtain some bounds for the difference of the average integral and left expression, also for the difference of the average integral and right expression in the inequality (1.1).

The class of $s$-convex functions in the third sense is defined on a special set given below, namely $p$-convex set.

Email address: zeynepeken@akdeniz.edu.tr (Z. Eken) 
Definition 1.1. [7] Let $U \subseteq \mathbb{R}^{n}$ and $0<p \leq 1$. If for each $x, y \in U, \lambda, \mu \geq 0$ such that $\lambda^{p}+\mu^{p}=1, \lambda x+\mu y \in U$, then $U$ is called a $p$-convex set in $\mathbb{R}^{n}$.

Not every convex set in $\mathbb{R}$ is $p$-convex. $p$-Convex sets are intervals that accept 0 as the boundary point. In line with [14] the concept of $p$-convex set given in Definition 1.1 is hereinafter referred to as $s$-convex set.

Definition 1.2. [14] Let $s \in(0,1]$ and $U \subseteq \mathbb{R}^{n}$ be a $s$-convex set. A function $f: U \rightarrow \mathbb{R}$ is said to be $s$-convex function in the third sense if the inequality

$$
f(\lambda x+\mu y) \leq \lambda^{\frac{1}{s}} f(x)+\mu^{\frac{1}{s}} f(y)
$$

is satisfied for all $x, y \in U$ and $\lambda, \mu \geq 0$ such that $\lambda^{s}+\mu^{s}=1$.

The inequality (1.2) can also be expressed in the following ways:

$$
f\left(\lambda^{\frac{1}{s}} x+(1-\lambda)^{\frac{1}{s}} y\right) \leq \lambda^{\frac{1}{s^{2}}} f(x)+(1-\lambda)^{\frac{1}{s^{2}}} f(y)
$$

or

$$
f\left(\lambda x+\left(1-\lambda^{s}\right)^{\frac{1}{s}} y\right) \leq \lambda^{\frac{1}{s}} f(x)+\left(1-\lambda^{s}\right)^{\frac{1}{s^{2}}} f(y)
$$

where $\lambda \in[0,1]$ and $x, y \in U$. The class of these functions is denoted by $K_{s}^{3}$.

An example of $s$-convex functions in the third sense is given below.

Example 1.3. [14] Let $s \in(0,1]$ and $a, b, c \in \mathbb{R}$ with $b<0$ and $a, c \leq 0$. The function

$$
f(x)= \begin{cases}a, & \text { if } x=0 \\ b x^{\frac{1}{s}}+c, & \text { if } x>0\end{cases}
$$

is $s$-convex function in the third sense on $(0, \infty)$. By adding extra condition $c \leq a$, we can say $f$ is $s$-convex function in the third sense on $[0, \infty)$.

Not every convex function is an $s$-convex function in the third sense. Although the function $f(x)=-x^{2}$ on $(0, \infty)$ is not convex, it is $s$-convex function in the third sense. Similarly, not every $s$-convex function in the third sense is a convex function. Although the function $f(x)=e^{x}$ on $(0, \infty)$ is a convex function, it is not an $s$-convex function in the third sense.

A Hermite-Hadamard type inequality for the $s$-convex function in the third sense is given in the following theorem.

Theorem 1.4. [24] Let $f: \mathbb{R}_{+} \rightarrow \mathbb{R}_{+}$be an integrable s-convex function in the third sense. For $a, b \in \mathbb{R}_{+}$with $a<b$, the following inequality holds

$$
2^{\frac{1}{s^{2}}-1} f\left(\frac{a+b}{2^{\frac{1}{s}}}\right) \leq \int_{a}^{b} f(x) d x \leq \frac{1}{(s+1)}\left(\frac{1}{s}[f(a) b+f(b) a] B\left(\frac{1}{s^{2}}, \frac{1}{s}\right)+s[f(b) b+f(a) a]\right)
$$

where the function B is the Beta function defined as

$$
B(x, y)=\int_{0}^{1} t^{x-1}(1-t)^{y-1} d t
$$

where $x, y>0$.

\section{Main Results}

By using the following lemma, we will determine an upper bound for the right side of Hermite-Hadamard inequality for $s$-convex functions in the third sense:

Lemma 2.1. Let $a, b \in \mathbb{R}$ with $a<b, f:[a, b] \subseteq \mathbb{R} \rightarrow \mathbb{R}$ be a differentiable function. If $f^{\prime}$ is integrable on $[a, b]$, then the following equality holds:

$$
\begin{aligned}
\frac{f(a)+f(b)}{2}-\frac{1}{b-a} \int_{a}^{b} f(x) d x= & \frac{1}{2 s(a-b)} \int_{0}^{1}\left[a+b-2\left(t^{\frac{1}{s}} b+(1-t)^{\frac{1}{s}} a\right)\right] f^{\prime}\left(t^{\frac{1}{s}} b+(1-t)^{\frac{1}{s}} a\right) \\
& \cdot\left[t^{\frac{1}{s}-1} b-(1-t)^{\frac{1}{s}-1} a\right] d t .
\end{aligned}
$$


Proof. Using the partial integration formula and changing the variable as $x=t^{\frac{1}{s}} b+(1-t)^{\frac{1}{s}} a$, we get the desired equality as follows:

$$
\begin{aligned}
& \frac{1}{2 s(a-b)} \int_{0}^{1}\left[a+b-2\left(t^{\frac{1}{s}} b+(1-t)^{\frac{1}{s}} a\right)\right] f^{\prime}\left(t^{\frac{1}{s}} b+(1-t)^{\frac{1}{s}} a\right)\left[t^{\frac{1}{s}-1} b-(1-t)^{\frac{1}{s}-1} a\right] d t \\
&=\left.\frac{1}{2(a-b)}\left[a+b-2\left(t^{\frac{1}{s}} b+(1-t)^{\frac{1}{s}} a\right)\right] f\left(t^{\frac{1}{s}} b+(1-t)^{\frac{1}{s}} a\right)\right|_{0} ^{1} \\
&+\frac{1}{s(a-b)} \int_{0}^{1} f\left(t^{\frac{1}{s}} b+(1-t)^{\frac{1}{s}} a\right)\left[t^{\frac{1}{s}-1} b-(1-t)^{\frac{1}{s}-1} a\right] d t \\
&= \frac{f(a)+f(b)}{2}-\frac{1}{b-a} \int_{a}^{b} f(x) d x .
\end{aligned}
$$

Theorem 2.2. Let $a, b \in \mathbb{R}$ with $a<b$ and $f: \mathbb{R} \rightarrow \mathbb{R}$ be differentiable function such that $\left|f^{\prime}\right|$ is integrable on $[a, b]$ and s-convex function in the third sense on $\mathbb{R}$. Then the following inequality holds:

$$
\left|\frac{f(a)+f(b)}{2}-\frac{1}{b-a} \int_{a}^{b} f(x) d x\right| \leq \frac{3 s}{2\left(s^{2}+1\right)(b-a)}(|a|+|b|)^{2}\left(\left|f^{\prime}(a)\right|+\left|f^{\prime}(b)\right|\right)
$$

Proof. Using Lemma 2.1, triangle inequality and the $s$-convexity of $\left|f^{\prime}\right|$, we have

$$
\begin{aligned}
& \left|\frac{f(a)+f(b)}{2}-\frac{1}{b-a} \int_{a}^{b} f(x) d x\right| \\
& \quad \leq \frac{1}{2 s(b-a)} \int_{0}^{1}\left|a+b-2\left(t^{\frac{1}{s}} b+(1-t)^{\frac{1}{s}} a\right)\right|\left|f^{\prime}\left(t^{\frac{1}{s}} b+(1-t)^{\frac{1}{s}} a\right)\right|\left|t^{\frac{1}{s}-1} b-(1-t)^{\frac{1}{s}-1} a\right| d t \\
& \quad \leq \frac{1}{2 s(b-a)} \int_{0}^{1}\left|a+b-2\left(t^{\frac{1}{s}} b+(1-t)^{\frac{1}{s}} a\right)\right|\left|t^{\frac{1}{s}-1} b-(1-t)^{\frac{1}{s}-1} a\right|\left(t^{\frac{1}{s^{2}}}\left|f^{\prime}(b)\right|+(1-t)^{\frac{1}{s^{2}}}\left|f^{\prime}(a)\right|\right) d t \\
& \quad \leq \frac{1}{2 s(b-a)} 3(|a|+|b|)(|a|+|b|)\left(\int_{0}^{1} t^{\frac{1}{s^{2}}}\left|f^{\prime}(b)\right| d t+\int_{0}^{1}(1-t)^{\frac{1}{s^{2}}}\left|f^{\prime}(a)\right| d t\right) \\
& \quad=\frac{3 s}{2\left(s^{2}+1\right)(b-a)}(|a|+|b|)^{2}\left(\left|f^{\prime}(a)\right|+\left|f^{\prime}(b)\right|\right) .
\end{aligned}
$$

It is clear that the sharper versions for the inequality (2.1) can be obtained. It is given in the following theorems three sharper versions for this inequality:

Theorem 2.3. Let $a, b \in \mathbb{R}$ with $a<b$ and $f: \mathbb{R} \rightarrow \mathbb{R}$ be differentiable function such that $\left|f^{\prime}\right|$ is integrable on $[a, b]$ and s-convex function in the third sense on $\mathbb{R}$. Then the following inequality holds:

$$
\begin{aligned}
&\left|\frac{f(a)+f(b)}{2}-\frac{1}{b-a} \int_{a}^{b} f(x) d x\right| \\
& \leq \frac{s}{2(a-b)}\left[\frac{1}{s+1}(|a|+|b|)\left(\left|f^{\prime}(b)\right| b+\left|f^{\prime}(a)\right| a\right)+\frac{2}{2 s+1}\left(\left|f^{\prime}(b)\right| b^{2}+\left|f^{\prime}(a)\right| a^{2}\right)\right] \\
&+\frac{1}{2 s(s+1)(b-a)}(|a|+|b|)\left(\left|a f^{\prime}(b)\right|+\left|b f^{\prime}(a)\right|\right) B\left(\frac{1}{s}, \frac{1}{s^{2}}\right) \\
&+\frac{1}{s(2 s+1)(b-a)}\left(\left|f^{\prime}(b)\right| a^{2}+\left|f^{\prime}(a)\right| b^{2}\right) B\left(\frac{2}{s}, \frac{1}{s^{2}}\right) \\
&+\frac{1}{s(b-a)}|a b|\left(\left|f^{\prime}(b)\right|+\left|f^{\prime}(a)\right|\right) B\left(\frac{1}{s}+\frac{1}{s^{2}}, \frac{1}{s}\right) .
\end{aligned}
$$


Proof. If we put $g(t):=t^{\frac{1}{s}-1} b-(1-t)^{\frac{1}{s}-1} a$ and $h(t):=a+b-2\left(t^{\frac{1}{s}} b+(1-t)^{\frac{1}{s}} a\right)$, then we have

Using triangle inequality, we have

$$
\begin{aligned}
\left|\frac{f(a)+f(b)}{2}-\frac{1}{b-a} \int_{a}^{b} f(x) d x\right| & \leq \frac{1}{2 s(b-a)} \int_{0}^{1}|h(t) g(t)|\left|f^{\prime}\left(t^{\frac{1}{s}} b+(1-t)^{\frac{1}{s}} a\right)\right| d t \\
& \leq \frac{1}{2 s(b-a)} \int_{0}^{1}|h(t) g(t)|\left(t^{\frac{1}{s^{2}}}\left|f^{\prime}(b)\right|+(1-t)^{\frac{1}{s^{2}}}\left|f^{\prime}(a)\right|\right) d t .
\end{aligned}
$$

$$
\begin{aligned}
& |h(t) g(t)| \\
& =\left|\left(a b+b^{2}\right) t^{\frac{1}{s}-1}-\left(a^{2}+a b\right)(1-t)^{\frac{1}{s}-1}-2 b^{2} t^{\frac{2}{s}-1}+2 a b\left(t^{\frac{1}{s}}(1-t)^{\frac{1}{s}-1}-t^{\frac{1}{s}-1}(1-t)^{\frac{1}{s}}\right)+2 a^{2}(1-t)^{\frac{2}{s}-1}\right| \\
& \leq\left(|a b|+b^{2}\right) t^{\frac{1}{s}-1}+\left(a^{2}+|a b|\right)(1-t)^{\frac{1}{s}-1}+2 b^{2} t^{\frac{2}{s}-1}+2|a b|\left(t^{\frac{1}{s}}(1-t)^{\frac{1}{s}-1}+t^{\frac{1}{s}-1}(1-t)^{\frac{1}{s}}\right)+2 a^{2}(1-t)^{\frac{2}{s}-1} .
\end{aligned}
$$

Multiplying (2.4) with $\left(t^{\frac{1}{s^{2}}}\left|f^{\prime}(b)\right|+(1-t)^{\frac{1}{s^{2}}}\left|f^{\prime}(a)\right|\right)$, expanding and integrating on [0,1] with respect to $t$ and using properties of the Beta function $B$, we get

$$
\begin{aligned}
& \int_{0}^{1}|h(t) g(t)|\left(t^{\frac{1}{s^{2}}}\left|f^{\prime}(b)\right|+(1-t)^{\frac{1}{s^{2}}}\left|f^{\prime}(a)\right|\right) d t \\
& \leq \frac{s^{2}}{s+1}(|a|+|b|)\left(\left|f^{\prime}(b)\right||b|+\left|f^{\prime}(a)\right||a|\right)+\frac{2 s^{2}}{2 s+1}\left(\left|f^{\prime}(b)\right| b^{2}+\left|f^{\prime}(a)\right| a^{2}\right) \\
&+\frac{1}{s+1}(|a|+|b|)\left(\left|a f^{\prime}(b)\right|+\left|b f^{\prime}(a)\right|\right) B\left(\frac{1}{s}, \frac{1}{s^{2}}\right) \\
&+\frac{2}{2 s+1}\left(\left|f^{\prime}(b)\right| a^{2}+\left|f^{\prime}(a)\right| b^{2}\right) B\left(\frac{2}{s}, \frac{1}{s^{2}}\right) \\
&+2|a b|\left(\left|f^{\prime}(b)\right|+\left|f^{\prime}(a)\right|\right) B\left(\frac{1}{s}+\frac{1}{s^{2}}, \frac{1}{s}\right) .
\end{aligned}
$$

When this inequality is used in the inequality (2.3), (2.2) is obtained.

Theorem 2.4. Let $a, b \in \mathbb{R}$ with $a<b$ and $f: \mathbb{R} \rightarrow \mathbb{R}$ be differentiable function such that $\left|f^{\prime}\right|$ is integrable on $[a, b]$ and s-convex function in the third sense on $\mathbb{R}$. Then the following inequality holds:

$$
\begin{aligned}
& \left|\frac{f(a)+f(b)}{2}-\frac{1}{b-a} \int_{a}^{b} f(x) d x\right| \\
& \quad \leq \frac{s}{2\left(s^{2}+1\right)(b-a)} \max \left\{|g(0)|,|g(1)|,\left|g\left(t_{1}\right)\right|\right\} \cdot \max \left\{|h(0)|,|h(1)|,\left|h\left(t_{2}\right)\right|\right\}\left(\left|f^{\prime}(a)\right|+\left|f^{\prime}(b)\right|\right)
\end{aligned}
$$

where

$$
g(t)=t^{\frac{1}{s}-1} b-(1-t)^{\frac{1}{s}-1} a \text { and } h(t)=a+b-2\left(t^{\frac{1}{s}} b+(1-t)^{\frac{1}{s}} a\right)
$$

as in the proof of the above theorem and for $a \neq 0$

$$
t_{1}=\left\{\begin{array}{ccc}
\left(1+\left|\frac{b}{a}\right|^{\frac{s}{1-2 s}}\right)^{-1} & \text { if } & s \neq \frac{1}{2} \\
0 \text { or } 1 & \text { if } & s=\frac{1}{2}
\end{array}, \quad t_{2}=\left\{\begin{array}{ccc}
\left(1+\left|\frac{b}{a}\right|^{\frac{s}{1-s}}\right)^{-1} & \text { if } & s \neq 1 \\
0 & \text { if } & s=1
\end{array},\right.\right.
$$

for $a=0, t_{1}, t_{2}$ equal to 0 or 1 .

Proof. Let $s \neq 1$ and $s \neq \frac{1}{2}$. From Lemma 2.1, as in the proof of Theorem 2.2, we have

$$
\left|\frac{f(a)+f(b)}{2}-\frac{1}{b-a} \int_{a}^{b} f(x) d x\right| \leq \frac{1}{2 s(b-a)} \int_{0}^{1}|h(t) g(t)|\left(t^{\frac{1}{s^{2}}}\left|f^{\prime}(b)\right|+(1-t)^{\frac{1}{s^{2}}}\left|f^{\prime}(a)\right|\right) d t
$$

where

$$
g(t)=t^{\frac{1}{s}-1} b-(1-t)^{\frac{1}{s}-1} a, \quad h(t)=a+b-2\left(t^{\frac{1}{s}} b+(1-t)^{\frac{1}{s}} a\right) .
$$

Let $a \neq 0$. In search of extremum points of $g$ and $h$ it is seen that while $\frac{b}{a}<0$ and $\frac{b}{a}>0, g$ and $h$ have one extremum point in [0,1], i.e. $g$ and $h$ are unimodal functions on [0,1], respectively. In other cases $g$ and $h$ will be monotone 
functions. So $g$ and $h$ take extremum values either at the points $t_{1}$ and $t_{2}$ for proper values of $a, b$, respectively, or at the points $t=0$ or $t=1$ in common.

If we take $\left|\frac{b}{a}\right|$ in the expression of $t_{1}$ and $t_{2}$, we can express the largest values that can be reached in the $[0,1]$ interval, regardless of the sign of $\frac{b}{a}$ as follows.

Thus

$$
|g(t)| \leq \max \left\{|g(0)|,|g(1)|,\left|g\left(t_{1}\right)\right|\right\}
$$

and

$$
|h(t)| \leq \max \left\{|h(0)|,|h(1)|,\left|h\left(t_{2}\right)\right|\right\}
$$

is derived.

For the case $a=0$, extremum values are obtained for $t=0, t=1$, which is included in the inequality above.

If $s=1$, in this case, $h(t)=(1-2 t)(b-a)$ is a linear decreasing function and it attains extremum values at $t=0$ or $t=1$. Also, although $t_{2}$ is undefined for $s=1$, we can calculate $t_{2}$ when $s \rightarrow 1^{-}$. Taking into consideration that $\left|\frac{b}{a}\right|>1$, we have

$$
t_{2}=\lim _{s \rightarrow 1^{-}}\left(1+\left|\frac{b}{a}\right|^{\frac{s}{1-s}}\right)^{-1}=0
$$

This means that in case $s=1,(2.6)$ is true.

If $s=\frac{1}{2}$, in this case, $g(t)=t(b+a)-a$ is a linear monotone function and it attains extremum values at $t=0$ or $t=1$. Also, although $t_{1}$ is undefined for $s=\frac{1}{2}$, we can calculate $t_{1}$ when $s \rightarrow \frac{1}{2}$. Taking into consideration that $\left|\frac{b}{a}\right|>1$, we have

$$
\lim _{s \rightarrow \frac{1^{+}}{2}}\left(1+\left|\frac{b}{a}\right|^{\frac{s}{1-2 s}}\right)^{-1}=1 \text { and } \lim _{s \rightarrow \frac{1}{2}^{-}}\left(1+\left|\frac{b}{a}\right|^{\frac{s}{1-2 s}}\right)^{-1}=0 .
$$

Whichever of the limits is used as $t_{1}$, it makes (2.5) true.

Thus, in a similar way in the proof of Theorem 2.2, by using the $s$-convexity of $\left|f^{\prime}\right|$, we get the desired result for each $s \in(0,1]$.

By making use of the Hölder inequality, some kind of extensions of the above theorems can be obtained as in the following theorems.

Theorem 2.5. Let $a, b \in \mathbb{R}$ with $a<b, p \in(1, \infty), f: \mathbb{R} \rightarrow \mathbb{R}$ be differentiable function such that $\left|f^{\prime}\right|^{p}$ is integrable on $[a, b]$ and s-convex function in the third sense on $\mathbb{R}$. Then the following inequality holds:

$$
\left|\frac{f(a)+f(b)}{2}-\frac{1}{b-a} \int_{a}^{b} f(x) d x\right| \leq \frac{3}{2 s(b-a)}\left(\frac{s^{2}}{s^{2}+1}\right)^{\frac{1}{p}}(|a|+|b|)^{2}\left(\left|f^{\prime}(a)\right|^{p}+\left|f^{\prime}(b)\right|^{p}\right)^{\frac{1}{p}} .
$$

Proof. Using Lemma 2.1, triangle and Hölder inequality and the $s$-convexity of $\left|f^{\prime}\right|^{p}$, we have

$$
\begin{aligned}
& \left|\frac{f(a)+f(b)}{2}-\frac{1}{b-a} \int_{a}^{b} f(x) d x\right| \\
& \leq \frac{1}{2 s(b-a)} \int_{0}^{1}\left|a+b-2\left(t^{\frac{1}{s}} b+(1-t)^{\frac{1}{s}} a\right)\right|\left|t^{\frac{1}{s}-1} b-(1-t)^{\frac{1}{s}-1} a\right|\left|f^{\prime}\left(t^{\frac{1}{s}} b+(1-t)^{\frac{1}{s}} a\right)\right| d t \\
& \left.=\frac{1}{2 s(b-a)}\left(\int_{0}^{1}\left(\mid a+b-2 t^{\frac{1}{s}} b-2(1-t)^{\frac{1}{s}} a\right)|| t^{\frac{1}{s}-1} b-(1-t)^{\frac{1}{s}-1} a \mid\right)^{\frac{p}{p-1}} d t\right)^{\frac{p-1}{p}}\left(\int_{0}^{1}\left|f^{\prime}\left(t^{\frac{1}{s}} b+(1-t)^{\frac{1}{s}} a\right)\right|^{p} d t\right)^{\frac{1}{p}} \\
& \leq \frac{1}{2 s(b-a)}\left(\int_{0}^{1}(|a|+|b|+2|b|+2|a|)^{\frac{p}{p-1}}(|b|+|a|)^{\frac{p}{p-1}} d t\right)^{\frac{p-1}{p}}\left(\int_{0}^{1}\left(t^{\frac{1}{s^{2}}}\left|f^{\prime}(b)\right|^{p}+(1-t)^{\frac{1}{s^{2}}}\left|f^{\prime}(a)\right|^{p}\right) d t\right)^{\frac{1}{p}} \\
& \leq \frac{3}{2 s(b-a)}\left(\frac{s^{2}}{s^{2}+1}\right)^{\frac{1}{p}}(|a|+|b|)^{2}\left(\left|f^{\prime}(a)\right|^{p}+\left|f^{\prime}(b)\right|^{p}\right)^{\frac{1}{p}} .
\end{aligned}
$$


Theorem 2.6. Let $a, b \in \mathbb{R}$ with $a<b$ and $f: \mathbb{R} \rightarrow \mathbb{R}$ be differentiable function such that $\left|f^{\prime}\right|^{p}$ is integrable on $[a, b]$ with $p>1$ and s-convex function in the third sense on $\mathbb{R}$. Then the following inequality holds:

$$
\begin{aligned}
& \left|\frac{f(a)+f(b)}{2}-\frac{1}{b-a} \int_{a}^{b} f(x) d x\right| \\
& \quad \leq \frac{1}{2 s(b-a)}\left(\frac{s^{2}}{s^{2}+1}\right)^{\frac{1}{p}} \max \left\{|g(0)|,|g(1)|,\left|g\left(t_{1}\right)\right|\right\} \cdot \max \left\{|h(0)|,|h(1)|,\left|h\left(t_{2}\right)\right|\right\}\left(\left|f^{\prime}(a)\right|^{p}+\left|f^{\prime}(b)\right|^{p}\right)^{\frac{1}{p}}
\end{aligned}
$$

where $g, h$ and $t_{1}, t_{2}$ are defined as in Theorem 2.4.

Proof. By applying the Hölder inequality as in the proof of Theorem 2.5, then using the findings about the maximum of $h$ and $g$ from the proof of Theorem 2.4, the desired inequality is obtained.

By using the following lemma, we will determine an upper bound for the left side of Hermite-Hadamard inequality for $s$-convex functions in the third sense:

Lemma 2.7. Let $a, b \in \mathbb{R}$ with $a<b, f:[a, b] \subseteq \mathbb{R} \rightarrow \mathbb{R}$ be a differentiable function. If $f^{\prime}$ is integrable on $[a, b]$, then the following equality holds:

$$
\begin{aligned}
f( & \left.\frac{a+b}{2}\right)-\frac{1}{b-a} \int_{a}^{b} f(x) d x \\
= & \frac{1}{s(b-a)} \int_{0}^{1}\left[t^{\frac{1}{s}} \frac{a+b}{2}+\left((1-t)^{\frac{1}{s}}-1\right) a\right] f^{\prime}\left(t^{\frac{1}{s}} \frac{a+b}{2}+(1-t)^{\frac{1}{s}} a\right)\left[t^{\frac{1}{s}-1} \frac{a+b}{2}-(1-t)^{\frac{1}{s}-1} a\right] d t \\
& +\frac{1}{s(b-a)} \int_{0}^{1}\left[b\left(t^{\frac{1}{s}}-1\right)+(1-t)^{\frac{1}{s}} \frac{a+b}{2}\right] f^{\prime}\left(t^{\frac{1}{s}} b+(1-t)^{\frac{1}{s}} \frac{a+b}{2}\right)\left[t^{\frac{1}{s}-1} b-(1-t)^{\frac{1}{s}-1} \frac{a+b}{2}\right] d t .
\end{aligned}
$$

Proof. If we apply partial integration to the integrals on the right side of equality (2.7) and make the necessary variable substitution, we get

$$
\begin{aligned}
& \frac{1}{s(b-a)} \int_{0}^{1}\left[t^{\frac{1}{s}} \frac{a+b}{2}+\left((1-t)^{\frac{1}{s}}-1\right) a\right] f^{\prime}\left(t^{\frac{1}{s}} \frac{a+b}{2}+(1-t)^{\frac{1}{s}} a\right)\left[t^{\frac{1}{s}-1} \frac{a+b}{2}-(1-t)^{\frac{1}{s}-1} a\right] d t \\
& +\frac{1}{s(b-a)} \int_{0}^{1}\left[b\left(t^{\frac{1}{s}}-1\right)+(1-t)^{\frac{1}{s}} \frac{a+b}{2}\right] f^{\prime}\left(t^{\frac{1}{s}} b+(1-t)^{\frac{1}{s}} \frac{a+b}{2}\right)\left[t^{\frac{1}{s}-1} b-(1-t)^{\frac{1}{s}-1} \frac{a+b}{2}\right] d t \\
& =\frac{1}{(b-a)}\left[\left(\frac{a+b}{2}-a\right) f\left(\frac{a+b}{2}\right)-\int_{a}^{\frac{a+b}{2}} f(x) d x\right]+\frac{1}{(b-a)}\left[\left(b-\frac{a+b}{2}\right) f\left(\frac{a+b}{2}\right)-\int_{\frac{a+b}{2}}^{b} f(x) d x\right] \\
& =f\left(\frac{a+b}{2}\right)-\frac{1}{b-a} \int_{a}^{b} f(x) d x .
\end{aligned}
$$

Theorem 2.8. Let $a, b \in \mathbb{R}$ with $a<b$ and $f: \mathbb{R} \rightarrow \mathbb{R}$ be differentiable function such that $\left|f^{\prime}\right|$ is integrable on $[a, b]$ and s-convex function in the third sense on $\mathbb{R}$. Then the following inequality holds:

$$
\begin{aligned}
& \left|f\left(\frac{a+b}{2}\right)-\frac{1}{b-a} \int_{a}^{b} f(x) d x\right| \\
& \quad \leq \frac{s}{\left(s^{2}+1\right)(b-a)}\left[\left(\frac{3|a|+|b|}{2}\right)^{2}\left|f^{\prime}(a)\right|+\frac{5 a^{2}+6|a b|+5 b^{2}}{2}\left|f^{\prime}\left(\frac{a+b}{2}\right)\right|+\left(\frac{|a|+3|b|}{2}\right)^{2}\left|f^{\prime}(b)\right|\right] .
\end{aligned}
$$


Proof. From Lemma 2.7, triangle inequality and the $s$-convexity of $\left|f^{\prime}\right|$, we have

$$
\begin{aligned}
&\left|f\left(\frac{a+b}{2}\right)-\frac{1}{b-a} \int_{a}^{b} f(x) d x\right| \\
& \leq \frac{1}{s(b-a)} \int_{0}^{1}\left|t^{\frac{1}{s}} \frac{a+b}{2}+\left((1-t)^{\frac{1}{s}}-1\right) a\right|\left|t^{\frac{1}{s}-1} \frac{a+b}{2}-(1-t)^{\frac{1}{s}-1} a\right|\left|f^{\prime}\left(t^{\frac{1}{s}} \frac{a+b}{2}+(1-t)^{\frac{1}{s}} a\right)\right| d t \\
& \quad+\frac{1}{s(b-a)} \int_{0}^{1}\left|b\left(t^{\frac{1}{s}}-1\right)+(1-t)^{\frac{1}{s}} \frac{a+b}{2}\right|\left|t^{\frac{1}{s}-1} b-(1-t)^{\frac{1}{s}-1} \frac{a+b}{2}\right|\left|f^{\prime}\left(t^{\frac{1}{s}} b+(1-t)^{\frac{1}{s}} \frac{a+b}{2}\right)\right| d t \\
& \leq \frac{1}{s(b-a)} \int_{0}^{1}\left(\frac{|a|+|b|}{2}+|a|\right)^{2}\left(t^{\frac{1}{s^{2}}}\left|f^{\prime}\left(\frac{a+b}{2}\right)\right|+(1-t)^{\frac{1}{s^{2}}}\left|f^{\prime}(a)\right|\right) d t \\
&+\frac{1}{s(b-a)} \int_{0}^{1}\left(\frac{|a|+|b|}{2}+|b|\right)^{2}\left(t^{\frac{1}{s^{2}}}\left|f^{\prime}(b)\right|+(1-t)^{\frac{1}{s^{2}}}\left|f^{\prime}\left(\frac{a+b}{2}\right)\right|\right) d t \\
& \leq \frac{s}{\left(s^{2}+1\right)(b-a)}\left[\left(\frac{3|a|+|b|}{2}\right)^{2}\left|f^{\prime}(a)\right|+\frac{5 a^{2}+6|a b|+5 b^{2}}{2}\left|f^{\prime}\left(\frac{a+b}{2}\right)\right|+\left(\frac{|a|+3|b|}{2}\right)^{2}\left|f^{\prime}(b)\right|\right]
\end{aligned}
$$

Theorem 2.9. Let $a, b \in \mathbb{R}_{++}$with $a<b$ and $f: \mathbb{R}_{++} \rightarrow \mathbb{R}$ be differentiable function such that $\left|f^{\prime}\right|$ is integrable on $[a, b]$ and s-convex function in the third sense on $\mathbb{R}$. Let

$$
\begin{aligned}
& g_{1}(t)=t^{\frac{1}{s}} \frac{a+b}{2}+\left((1-t)^{\frac{1}{s}}-1\right) a \text { and } g_{2}(t)=b\left(t^{\frac{1}{s}}-1\right)+(1-t)^{\frac{1}{s}} \frac{a+b}{2} \\
& h_{1}(t)=t^{\frac{1}{s}-1} \frac{a+b}{2}-(1-t)^{\frac{1}{s}-1} a \text { and } h_{2}(t)=t^{\frac{1}{s}-1} b-(1-t)^{\frac{1}{s}-1} \frac{a+b}{2} .
\end{aligned}
$$

Then

$$
\left|f\left(\frac{a+b}{2}\right)-\frac{1}{b-a} \int_{a}^{b} f(x) d x\right| \leq \frac{1}{(s+1)(b-a)}\left(w_{1}\left|f^{\prime}(a)\right|+\left(w_{1}+w_{2}\right) f^{\prime}\left(\frac{a+b}{2}\right)+w_{2}\left|f^{\prime}(b)\right|\right)
$$

where

$$
\begin{aligned}
& w_{1}=\max \left\{\left|g_{1}(0)\right|,\left|g_{1}(1)\right|,\left|g_{1}\left(t_{1}\right)\right|\right\} \cdot \max \left\{\left|h_{1}(0)\right|,\left|h_{1}(1)\right|,\left|h_{1}\left(s_{1}\right)\right|\right\}, \\
& w_{2}=\max \left\{\left|g_{2}(0)\right|,\left|g_{2}(1)\right|,\left|g_{2}\left(t_{2}\right)\right|\right\} \cdot \max \left\{\left|h_{2}(0)\right|,\left|h_{2}(1)\right|,\left|h_{2}\left(s_{2}\right)\right|\right\}
\end{aligned}
$$

and

$$
\begin{aligned}
& t_{1}=\left\{\begin{array}{ccc}
\left(1+\left(\frac{a+b}{2 a}\right)^{\frac{s}{1-s}}\right)^{-1} & \text { if } & s \neq 1 \\
1 & \text { if } & s=1
\end{array}, \quad t_{2}=\left\{\begin{array}{ccc}
\left(1+\left(\frac{a+b}{2 b}\right)^{\frac{s}{s-1}}\right)^{-1} & \text { if } & s \neq 1 \\
1 & \text { if } & s=1
\end{array},\right.\right. \\
& s_{1}=\left\{\begin{array}{ccc}
\left(1+\left(-\frac{a+b}{2 a}\right)^{\frac{s}{1-2 s}}\right)^{-1} & \text { if } & s \neq \frac{1}{2} \\
0 \text { or } 1 & \text { if } \quad s=\frac{1}{2}
\end{array} \quad, \quad s_{2}=\left\{\begin{array}{cll}
\left(1+\left(-\frac{a+b}{2 b}\right)^{\frac{s}{2 s-1}}\right)^{-1} & \text { if } & s \neq \frac{1}{2} \\
0 \text { or } 1 & \text { if } & s=\frac{1}{2} .
\end{array} .\right.\right.
\end{aligned}
$$

Proof. Let $s \neq 1$ and $s \neq \frac{1}{2}$. According to first derivatives of functions $g_{1}, g_{2}, h_{1}, h_{2}$, it is understood that these functions are either monotonic or unimodal functions on $[0,1]$ with respect to values of $a, b, s$. The maximum values of $\left|g_{1}(t)\right|,\left|g_{2}(t)\right|,\left|h_{1}(t)\right|,\left|h_{2}(t)\right|$ are attained at either boundary points of [0,1] or extremum points. The extremum points are determined as $t_{1}, t_{2}, s_{1}, s_{2}$. In cases $s=1$ and $s=\frac{1}{2}$, the idea in the proof of Theorem 2.4 is used and indicated values in the statement of the theorem are obtained. Thus

$$
\left|g_{i}(t)\right| \leq \max \left\{\left|g_{i}(0)\right|,\left|g_{i}(1)\right|,\left|g_{i}\left(t_{i}\right)\right|\right\} \text { and }\left|h_{i}(t)\right| \leq \max \left\{\left|h_{i}(0)\right|,\left|h_{i}(1)\right|,\left|h_{i}\left(s_{i}\right)\right|\right\}
$$


for $i=1$, 2. From Lemma 2.7, we have

$$
\begin{aligned}
& \left|f\left(\frac{a+b}{2}\right)-\frac{1}{b-a} \int_{a}^{b} f(x) d x\right| \\
& \leq \frac{1}{s(b-a)} \int_{0}^{1}\left|g_{1}(t)\right|\left|h_{1}(t)\right|\left|f^{\prime}\left(t^{\frac{1}{s}} \frac{a+b}{2}+(1-t)^{\frac{1}{s}} a\right)\right| d t+\frac{1}{s(b-a)} \int_{0}^{1}\left|g_{2}(t)\right|\left|h_{2}(t)\right|\left|f^{\prime}\left(t^{\frac{1}{s}} b+(1-t)^{\frac{1}{s}} \frac{a+b}{2}\right)\right| d t \\
& \leq \frac{1}{s(b-a)} \int_{0}^{1} \max \left\{\left|g_{1}(0)\right|,\left|g_{1}(1)\right|,\left|g_{1}\left(t_{1}\right)\right|\right\} \max \left\{\left|h_{1}(0)\right|,\left|h_{1}(1)\right|,\left|h_{1}\left(s_{1}\right)\right|\right\}\left(t^{\frac{1}{s^{2}}}\left|f^{\prime}\left(\frac{a+b}{2}\right)\right|+(1-t)^{\frac{1}{s^{2}}}\left|f^{\prime}(a)\right|\right) d t \\
& +\frac{1}{s(b-a)} \int_{0}^{1} \max \left\{\left|g_{2}(0)\right|,\left|g_{2}(1)\right|,\left|g_{2}\left(t_{2}\right)\right|\right\} \max \left\{\left|h_{2}(0)\right|,\left|h_{2}(1)\right|,\left|h_{2}\left(s_{2}\right)\right|\right\}\left(t^{\frac{1}{s^{2}}}\left|f^{\prime}(b)\right|+(1-t)^{\frac{1}{s^{2}}}\left|f^{\prime}\left(\frac{a+b}{2}\right)\right|\right) d t \\
& \leq \frac{s}{\left(s^{2}+1\right)(b-a)}\left(w_{1}\left|f^{\prime}(a)\right|+\left(w_{1}+w_{2}\right) f^{\prime}\left(\frac{a+b}{2}\right)+w_{2}\left|f^{\prime}(b)\right|\right) .
\end{aligned}
$$

Theorem 2.10. Let $a, b \in \mathbb{R}$ with $a<b$ and $f: \mathbb{R} \rightarrow \mathbb{R}$ be differentiable function such that $\left|f^{\prime}\right|$ is integrable on $[a, b]$ and s-convex function in the third sense on $\mathbb{R}$. Then the following inequality holds:

$$
\begin{aligned}
\mid f\left(\frac{a+b}{2}\right) & -\frac{1}{b-a} \int_{a}^{b} f(x) d x \mid \\
\leq & \frac{s(3 s+2)}{(s+1)(2 s+1)(b-a)}\left(a^{2}\left|f^{\prime}(a)\right|+\left|f^{\prime}\left(\frac{a+b}{2}\right)\right|\left|\frac{a+b}{2}\right|(|a|+|b|)+b^{2}\left|f^{\prime}(b)\right|\right) \\
& +\frac{1}{s(s+1)(b-a)}\left(\left|f^{\prime}\left(\frac{a+b}{2}\right)\right|\left(a^{2}+b^{2}\right)+\left|\frac{a+b}{2}\right|\left(|a|\left|f^{\prime}(a)\right|+|b|\left|f^{\prime}(b)\right|\right)\right) B\left(\frac{1}{s}, \frac{1}{s^{2}}\right) \\
& +\frac{1}{s(2 s+1)(b-a)}\left(\left|f^{\prime}\left(\frac{a+b}{2}\right)\right|\left(a^{2}+b^{2}\right)+\left(\frac{a+b}{2}\right)^{2}\left(\left|f^{\prime}(a)\right|+\left|f^{\prime}(b)\right|\right)\right) B\left(\frac{2}{s}, \frac{1}{s^{2}}\right) \\
& +\frac{1}{s(b-a)}\left|\frac{a+b}{2}\right|\left(|a|\left|f^{\prime}(a)\right|+\left|f^{\prime}\left(\frac{a+b}{2}\right)\right|(|a|+|b|)+|b|\left|f^{\prime}(b)\right|\right) B\left(\frac{1}{s}, \frac{1}{s}+\frac{1}{s^{2}}\right) .
\end{aligned}
$$

Proof. Let $g_{1}, g_{2}, h_{1}$ and $h_{2}$ functions as in Theorem 2.9. Using triangle inequality, we have

$$
\begin{aligned}
& \left|g_{1}(t) h_{1}(t)\right|=\left|\left(t^{\frac{1}{s}} \frac{a+b}{2}+\left((1-t)^{\frac{1}{s}}-1\right) a\right)\left(t^{\frac{1}{s}-1} \frac{a+b}{2}-(1-t)^{\frac{1}{s}-1} a\right)\right| \\
& =\left|\left(\frac{a+b}{2}\right)^{2} t^{\frac{2}{s}-1}+a\left(\frac{a+b}{2}\right)\left(t^{\frac{1}{s}-1}(1-t)^{\frac{1}{s}}-t^{\frac{1}{s}-1}-t^{\frac{1}{s}}(1-t)^{\frac{1}{s}-1}\right)-a^{2}\left((1-t)^{\frac{2}{s}-1}+(1-t)^{\frac{1}{s}-1}\right)\right| \\
& \leq\left(\frac{a+b}{2}\right)^{2} t^{\frac{2}{s}-1}+|a|\left|\frac{a+b}{2}\right|\left(t^{\frac{1}{s}-1}(1-t)^{\frac{1}{s}}+t^{\frac{1}{s}-1}+t^{\frac{1}{s}}(1-t)^{\frac{1}{s}-1}\right)+a^{2}\left((1-t)^{\frac{2}{s}-1}+(1-t)^{\frac{1}{s}-1}\right) . \\
& \left|g_{2}(t) h_{2}(t)\right|=\left|\left(b\left(t^{\frac{1}{s}}-1\right)+(1-t)^{\frac{1}{s}} \frac{a+b}{2}\right)\left(t^{t^{\frac{1}{s}}-1} b-(1-t)^{\frac{1}{s}-1} \frac{a+b}{2}\right)\right| \\
& =\left|b^{2}\left(t^{\frac{2}{s}}-1-t^{\frac{1}{s}-1}\right)+b\left(\frac{a+b}{2}\right)\left(t^{\frac{1}{s}-1}(1-t)^{\frac{1}{s}}+(1-t)^{\frac{1}{s}-1}-t^{\frac{1}{s}}(1-t)^{\frac{1}{s}-1}\right)-\left(\frac{a+b}{2}\right)^{2}(1-t)^{\frac{2}{s}-1}\right| \\
& \leq b^{2}\left(t^{\frac{2}{s}-1}+t^{\frac{1}{s}-1}\right)+|b|\left|\frac{a+b}{2}\right|\left(t^{\frac{1}{s}-1}(1-t)^{\frac{1}{s}}+(1-t)^{\frac{1}{s}-1}+t^{\frac{1}{s}}(1-t)^{\frac{1}{s}-1}\right)+\left(\frac{a+b}{2}\right)^{2}(1-t)^{\frac{2}{s}-1} .
\end{aligned}
$$

If we multiply inequalities (2.8) and (2.9) with $\left(t^{\frac{1}{s^{2}}}\left|f^{\prime}\left(\frac{a+b}{2}\right)\right|+(1-t)^{\frac{1}{s^{2}}}\left|f^{\prime}(a)\right|\right)$ and $\left(t^{\frac{1}{s^{2}}}\left|f^{\prime}(b)\right|+(1-t)^{\frac{1}{s^{2}}}\left|f^{\prime}\left(\frac{a+b}{2}\right)\right|\right)$, respectively using Lemma 2.7 , integrating on $[0,1]$, using the $s$-convexity of $\left|f^{\prime}\right|$ and 
properties of the Beta function $B$, we have

$$
\begin{aligned}
\left|f\left(\frac{a+b}{2}\right)-\frac{1}{b-a} \int_{a}^{b} f(x) d x\right| & \leq \frac{1}{s(b-a)} \int_{0}^{1}\left|g_{1}(t) h_{1}(t)\right|\left(t^{\frac{1}{s^{2}}}\left|f^{\prime}\left(\frac{a+b}{2}\right)\right|+(1-t)^{\frac{1}{s^{2}}}\left|f^{\prime}(a)\right|\right) d t \\
& \left.+\frac{1}{s(b-a)} \int_{0}^{1}\left|g_{2}(t) h_{2}(t)\right|\left|t^{\frac{1}{s^{2}}}\right| f^{\prime}(b)\left|+(1-t)^{\frac{1}{s^{2}}}\right| f^{\prime}\left(\frac{a+b}{2}\right) \mid\right) d t \\
\leq & \frac{1}{s(b-a)} \int_{0}^{1}\left[\left(\frac{a+b}{2}\right)^{2} t^{\frac{2}{s}-1}+|a|\left|\frac{a+b}{2}\right|\left(t^{\frac{1}{s}-1}(1-t)^{\frac{1}{s}}+t^{\frac{1}{s}-1}+t^{\frac{1}{s}}(1-t)^{\frac{1}{s}-1}\right)+a^{2}\left((1-t)^{\frac{2}{s}-1}+(1-t)^{\frac{1}{s}-1}\right)\right] \\
& \cdot\left(t^{\frac{1}{s^{2}}}\left|f^{\prime}\left(\frac{a+b}{2}\right)\right|+(1-t)^{\frac{1}{s^{2}}}\left|f^{\prime}(a)\right|\right) d t \\
& +\frac{1}{s(b-a)} \int_{0}^{1}\left[b^{2}\left(t^{\frac{2}{s}-1}+t^{\frac{1}{s}-1}\right)+|b|\left|\frac{a+b}{2}\right|\left(t^{\frac{1}{s}-1}(1-t)^{\frac{1}{s}}+2(1-t)^{\frac{1}{s}-1}+t^{\frac{1}{s}}(1-t)^{\frac{1}{s}-1}\right)+\left(\frac{a+b}{2}\right)^{2}(1-t)^{\frac{2}{s}-1}\right] \\
& \cdot\left(t^{\frac{1}{s^{2}}}\left|f^{\prime}(b)\right|+(1-t)^{\frac{1}{s^{2}}}\left|f^{\prime}\left(\frac{a+b}{2}\right)\right|\right) d t \\
\leq & \frac{s(3 s+2)}{(s+1)(2 s+1)(b-a)}\left(a^{2}\left|f^{\prime}(a)\right|+\left|f^{\prime}\left(\frac{a+b}{2}\right)\right|\left|\frac{a+b}{2}\right|(|a|+|b|)+b^{2}\left|f^{\prime}(b)\right|\right) \\
& +\frac{1}{s(s+1)(b-a)}\left(\left|f^{\prime}\left(\frac{a+b}{2}\right)\right|\left(a^{2}+b^{2}\right)+\left|\frac{a+b}{2}\right|\left(|a|\left|f^{\prime}(a)\right|+|b|\left|f^{\prime}(b)\right|\right)\right) B\left(\frac{1}{s}, \frac{1}{s^{2}}\right) \\
& \left.+\frac{1}{s(2 s+1)(b-a)}\right) \\
& +\left|\frac{a+b}{s(b-a)}\right|\left(|a|\left|f^{\prime}\left(\frac{a+b}{2}\right)\right|\left(a^{2}+b^{2}\right)+\left(\frac{a+b}{2}\right)^{2}\left(\left|f^{\prime}(a)\right|+\left|f^{\prime}(b)\right|\right)\right) B\left(\frac{2}{s}, \frac{1}{s^{2}}\right)
\end{aligned}
$$

Theorem 2.11. Let $a, b \in \mathbb{R}$ with $a<b, p \in(1, \infty)$ and $f: \mathbb{R} \rightarrow \mathbb{R}$ be differentiable function such that $\left|f^{\prime}\right|^{p}$ is integrable on $[a, b]$ and s-convex function in the third sense on $\mathbb{R}$. Then the following inequality holds:

$$
\begin{aligned}
\left|f\left(\frac{a+b}{2}\right)-\frac{1}{b-a} \int_{a}^{b} f(x) d x\right| \leq & \frac{s}{\left(s^{2}+1\right)(b-a)}\left(2|a|+\left|\frac{a+b}{2}\right|\right)\left(|a|+\left|\frac{a+b}{2}\right|\right)\left(\left|f^{\prime}\left(\frac{a+b}{2}\right)\right|^{p}+\left|f^{\prime}(a)\right|^{p}\right)^{\frac{1}{p}} \\
& +\frac{s}{\left(s^{2}+1\right)(b-a)}\left(2|b|+\left|\frac{a+b}{2}\right|\right)\left(|b|+\left|\frac{a+b}{2}\right|\right)\left(\left|f^{\prime}\left(\frac{a+b}{2}\right)\right|^{p}+\left|f^{\prime}(b)\right|^{p}\right)^{\frac{1}{p}} .
\end{aligned}
$$

Proof. From Lemma 2.7, Hölder inequality, triangle inequality and the $s$-convexity of $\left|f^{\prime}\right|^{p}$, we have

$$
\begin{aligned}
\mid f\left(\frac{a+b}{2}\right) & -\frac{1}{b-a} \int_{a}^{b} f(x) d x \mid \\
\leq & \frac{1}{s(b-a)}\left(\int_{0}^{1}\left|g_{1}(t) h_{1}(t)\right|^{\frac{p}{p-1}} d t\right)^{\frac{p-1}{p}}\left(\int_{0}^{1}\left|f^{\prime}\left(t^{\frac{1}{s}} \frac{a+b}{2}+(1-t)^{\frac{1}{s}} a\right)\right|^{p} d t\right)^{\frac{1}{p}} \\
& +\frac{1}{s(b-a)}\left(\int_{0}^{1}\left|g_{2}(t) h_{2}(t)\right|^{\frac{p}{p-1}} d t\right)^{\frac{p-1}{p}}\left(\int_{0}^{1}\left|f^{\prime}\left(t^{\frac{1}{s}} b+(1-t)^{\frac{1}{s}} \frac{a+b}{2}\right)\right|^{p} d t\right)^{\frac{1}{p}} \\
\leq & \frac{1}{s(b-a)}\left(\int_{0}^{1} \mid\left(t^{\frac{1}{s}} \frac{a+b}{2}+\left((1-t)^{\frac{1}{s}}-1\right) a\right)\left(t^{\frac{1}{s}-1} \frac{a+b}{2}-(1-t)^{\frac{1}{s}-1} a\right)^{\frac{p-1}{p}} d t\right)^{\frac{p-1}{p}} \\
& \cdot\left(\int_{0}^{1}\left|f^{\prime}\left(t^{\frac{1}{s}} \frac{a+b}{2}+(1-t)^{\frac{1}{s}} a\right)\right|^{p} d t\right)^{\frac{1}{p}}
\end{aligned}
$$




$$
\begin{aligned}
& +\frac{1}{s(b-a)}\left(\int_{0}^{1}\left|\left(b\left(t^{\frac{1}{s}}-1\right)+(1-t)^{\frac{1}{s}} \frac{a+b}{2}\right)\left(t^{\frac{1}{s}-1} b-(1-t)^{\frac{1}{s}-1} \frac{a+b}{2}\right)\right|^{\frac{p}{p-1}} d t\right)^{\frac{p-1}{p}} \\
& \cdot\left(\int_{0}^{1}\left|f^{\prime}\left(t^{\frac{1}{s}} b+(1-t)^{\frac{1}{s}} \frac{a+b}{2}\right)\right|^{p} d t\right)^{\frac{1}{p}} \\
& \leq \frac{1}{s(b-a)}\left(\int_{0}^{1}\left(\left|\frac{a+b}{2}\right|+2|a|\right)^{\frac{p}{p-1}}\left(\left|\frac{a+b}{2}\right|+|a|\right)^{\frac{p}{p-1}} d t\right)^{\frac{p-1}{p}} \\
& \cdot\left(\int_{0}^{1}\left(t^{\frac{1}{s^{2}}}\left|f^{\prime}\left(\frac{a+b}{2}\right)\right|^{p}+(1-t)^{\frac{1}{s^{2}}}\left|f^{\prime}(a)\right|^{p}\right) d t\right)^{\frac{1}{p}} \\
& \left.+\frac{1}{s(b-a)}\left(\int_{0}^{1}\left(2|b|+\left|\frac{a+b}{2}\right|\right)\right)^{\frac{p}{p-1}}\left(|b|+\left|\frac{a+b}{2}\right|\right)^{\frac{p}{p-1}} d t\right)^{\frac{p-1}{p}} \\
& \cdot\left(\int_{0}^{1}\left(t^{\frac{1}{s^{2}}}\left|f^{\prime}(b)\right|^{p}+(1-t)^{\frac{1}{s^{2}}}\left|f^{\prime}\left(\frac{a+b}{2}\right)\right|^{p}\right) d t\right)^{\frac{1}{p}} \\
& \leq \frac{s}{\left(s^{2}+1\right)(b-a)}\left(2|a|+\left|\frac{a+b}{2}\right|\right)\left(|a|+\left|\frac{a+b}{2}\right|\right)\left(\left|f^{\prime}\left(\frac{a+b}{2}\right)\right|^{p}+\left|f^{\prime}(a)\right|^{p}\right)^{\frac{1}{p}} \\
& +\frac{s}{\left(s^{2}+1\right)(b-a)}\left(2|b|+\left|\frac{a+b}{2}\right|\right)\left(|b|+\left|\frac{a+b}{2}\right|\right)\left(\left|f^{\prime}\left(\frac{a+b}{2}\right)\right|^{p}+\left|f^{\prime}(b)\right|^{p}\right)^{\frac{1}{p}}
\end{aligned}
$$

Using the following lemma, we will determine an estimate of the integral of $s$-convex functions in the third sense as a Hermite-Hadamard type inequality:

Lemma 2.12. Let $a, b \in \mathbb{R}$ with $a<b, f:[a, b] \subseteq \mathbb{R} \rightarrow \mathbb{R}$ be a differentiable function. If $f^{\prime}$ is integrable on $[a, b]$, then the following equality holds:

$$
b f(b)-a f(a)-\int_{a}^{b} f(x) d x=\frac{1}{s} \int_{0}^{1}\left[b^{2} t^{t^{\frac{2}{s}}-1}-a^{2}(1-t)^{\frac{2}{s}-1}+a b t^{\frac{1}{s}-1}(1-t)^{\frac{1}{s}}-a b t^{\frac{1}{s}}(1-t)^{\frac{1}{s}-1}\right] f^{\prime}\left(t^{\frac{1}{s}} b+(1-t)^{\frac{1}{s}} a\right) d t .
$$

Proof. Applying the partial integration formula and changing the variable as $x=t^{\frac{1}{s}} b+(1-t)^{\frac{1}{s}} a$, then we have

$$
\begin{aligned}
& \frac{1}{s} \int_{0}^{1}\left[b^{2} t^{\frac{2}{s}-1}-a^{2}(1-t)^{\frac{2}{s}-1}+a b t^{\frac{1}{s}-1}(1-t)^{\frac{1}{s}}-a b t^{\frac{1}{s}}(1-t)^{\frac{1}{s}-1}\right] f^{\prime}\left(t^{\frac{1}{s}} b+(1-t)^{\frac{1}{s}} a\right) d t \\
& \quad=\int_{0}^{1}\left(t^{\frac{1}{s}} b+(1-t)^{\frac{1}{s}} a\right) f^{\prime}\left(t^{\frac{1}{s}} b+(1-t)^{\frac{1}{s}} a\right) \frac{1}{s}\left(t^{\frac{1}{s}-1} b-(1-t)^{\frac{1}{s}-1} a\right) d t \\
& =\left[\left(t^{\frac{1}{s}} b+(1-t)^{\frac{1}{s}} a\right) f\left(t^{\frac{1}{s}} b+(1-t)^{\frac{1}{s}} a\right)\right]_{0}^{1}-\int_{0}^{1} f\left(t^{\frac{1}{s}} b+(1-t)^{\frac{1}{s}} a\right) \frac{1}{s}\left(t^{\frac{1}{s}-1} b-(1-t)^{\frac{1}{s}-1} a\right) d t \\
& \quad=b f(b)-a f(a)-\int_{a}^{b} f(x) d x .
\end{aligned}
$$

Theorem 2.13. Let $a, b \in \mathbb{R}$ with $a<b$ and $f: \mathbb{R} \rightarrow \mathbb{R}$ be differentiable function such that $\left|f^{\prime}\right|$ is integrable on $[a, b]$ and s-convex function in the third sense on $\mathbb{R}$. Then the following inequality holds:

$$
\left|b f(b)-a f(a)-\int_{a}^{b} f(x) d x\right| \leq \frac{s}{s^{2}+1}(|a|+|b|)^{2}\left(\left|f^{\prime}(a)\right|+\left|f^{\prime}(b)\right|\right) .
$$


Proof. Using Lemma 2.12 and convexity of $\left|f^{\prime}\right|$, we obtain

$$
\begin{aligned}
& \left|b f(b)-a f(a)-\int_{a}^{b} f(x) d x\right| \\
& =\frac{1}{s}\left|\int_{0}^{1}\left[b^{2} t^{\frac{2}{s}-1}-a^{2}(1-t)^{\frac{2}{s}-1}+a b t^{\frac{1}{s}-1}(1-t)^{\frac{1}{s}}-a b t^{\frac{1}{s}}(1-t)^{\frac{1}{s}-1}\right] f^{\prime}\left(t^{\frac{1}{s}} b+(1-t)^{\frac{1}{s}} a\right) d t\right| \\
& \leq \frac{1}{s} \int_{0}^{1}\left|b^{2} t^{\frac{2}{s}-1}-a^{2}(1-t)^{\frac{2}{s}-1}+a b t^{\frac{1}{s}-1}(1-t)^{\frac{1}{s}}-a b t^{\frac{1}{s}}(1-t)^{\frac{1}{s}-1}\right|\left|f^{\prime}\left(t^{\frac{1}{s}} b+(1-t)^{\frac{1}{s}} a\right)\right| d t \\
& \leq \frac{1}{s} \int_{0}^{1}\left(\left|b^{2} t^{\frac{2}{s}-1}\right|+\left|a^{2}(1-t)^{\frac{2}{s}-1}\right|+\left|a b t^{\frac{1}{s}-1}(1-t)^{\frac{1}{s}}\right|+\left|a b t^{\frac{1}{s}}(1-t)^{\frac{1}{s}-1}\right|\right)\left(t^{\frac{1}{s^{2}}}\left|f^{\prime}(b)\right|+(1-t)^{\frac{1}{s^{2}}}\left|f^{\prime}(a)\right|\right) d t \\
& \leq \frac{1}{s} \int_{0}^{1}(|a|+|b|)^{2}\left(t^{\frac{1}{s^{2}}}\left|f^{\prime}(b)\right|+(1-t)^{\frac{1}{s^{2}}}\left|f^{\prime}(a)\right|\right) d t \\
& =\frac{s}{s^{2}+1}(|a|+|b|)^{2}\left(\left|f^{\prime}(a)\right|+\left|f^{\prime}(b)\right|\right) .
\end{aligned}
$$

Theorem 2.14. Let $f: \mathbb{R} \rightarrow \mathbb{R}$ be a differentiable function, $a, b \in \mathbb{R}$ with $a<b$ and $p \in(1, \infty)$ such that $\frac{1}{p}<s$. If $\left|f^{\prime}\right|^{p}$ is s-convex in the third sense on $\mathbb{R}$ then the following inequality holds:

$$
\left|b f(b)-a f(a)-\int_{a}^{b} f(x) d x\right| \leq \frac{1}{s}\left(\frac{s^{2}}{s^{2}+1}\right)^{\frac{1}{p}}(|a|+|b|)^{2}\left(\left|f^{\prime}(b)\right|^{p}+\left|f^{\prime}(a)\right|^{p}\right)^{\frac{1}{p}} .
$$

Proof. Using Lemma 2.12, Hölder inequality, triangle inequality and the $s$-convexity of $\left|f^{\prime}\right|^{p}$, we have

$$
\begin{aligned}
\mid b f(b) & -a f(a)-\int_{a}^{b} f(x) d x \mid \\
= & \frac{1}{s}\left|\int_{0}^{1}\left[b^{2} t^{\frac{2}{s}-1}-a^{2}(1-t)^{\frac{2}{s}-1}+a b t^{\frac{1}{s}-1}(1-t)^{\frac{1}{s}}-a b t^{\frac{1}{s}}(1-t)^{\frac{1}{s}-1}\right] f^{\prime}\left(t^{\frac{1}{s}} b+(1-t)^{\frac{1}{s}} a\right) d t\right| \\
\leq & \frac{1}{s}\left(\int_{0}^{1}\left|b^{2} t^{\frac{2}{s}-1}-a^{2}(1-t)^{\frac{2}{s}-1}+a b t^{\frac{1}{s}-1}(1-t)^{\frac{1}{s}}-a b t^{\frac{1}{s}}(1-t)^{\frac{1}{s}-1}\right|^{\frac{p}{p-1}} d t\right)^{\frac{p-1}{p}} \\
& \cdot\left(\int_{0}^{1}\left|f^{\prime}\left(t^{\frac{1}{s}} b+(1-t)^{\frac{1}{s}} a\right)\right|^{p} d t\right)^{\frac{1}{p}} \\
\leq & \frac{1}{s}\left(\int_{0}^{1}\left[b^{2} t^{\frac{2}{s}-1}+a^{2}(1-t)^{\frac{2}{s}-1}+|a b| t^{\frac{1}{s}-1}(1-t)^{\frac{1}{s}}+|a b| t^{\frac{1}{s}}(1-t)^{\frac{1}{s}-1}\right]^{\frac{p}{p-1}} d t\right)^{\frac{p-1}{p}} \\
& \cdot\left(\int_{0}^{1} t^{\frac{1}{s^{2}}}\left|f^{\prime}(b)\right|^{p}+(1-t)^{\frac{1}{s^{2}}}\left|f^{\prime}(a)\right|^{p} d t\right)^{\frac{1}{p}} \\
\leq & \frac{1}{s}\left(\int_{0}^{1}\left[b^{2}+2|a b|+a^{2}\right]^{\frac{p}{p-1}} d t\right)^{\frac{p-1}{p}}\left(\int_{0}^{1} t t^{\frac{1}{s^{2}}}\left|f^{\prime}(b)\right|^{p}+(1-t)^{\frac{1}{s^{2}}}\left|f^{\prime}(a)\right|^{p} d t\right)^{\frac{1}{p}} \\
= & \frac{1}{s}\left(\frac{s^{2}}{s^{2}+1}\right)^{\frac{1}{p}}(|a|+|b|)^{2}\left(\left|f^{\prime}(b)\right|^{p}+\left|f^{\prime}(a)\right|^{p}\right)^{\frac{1}{p}} .
\end{aligned}
$$




\section{Applications}

Using some of the results given above, we can find an upper bound for error in numerical integration for the functions whose absolute value of first derivatives are $s$-convex in the third sense via composite trapezoid rule.

Let $f$ be an integrable function on $[a, b]$ and $P$ be a partition of the interval $[a, b]$, i.e. $P: a=x_{0}<x_{1}<\cdots<$ $x_{n-1}<x_{n}=b$ and $\Delta x_{i+1}=x_{i+1}-x_{i}$. Then

$$
\int_{a}^{b} f(x) d x=\sum_{k=0}^{n-1} \frac{f\left(x_{k}\right)+f\left(x_{k+1}\right)}{2} \Delta x_{k+1}+E(f, P)
$$

where $E(f, P)$ is called the error of integral with respect to $P$. There are some ways to estimate an upper bound for $E(f, P)$. For $s$-convex functions in the third sense we suggest the following proposition:

Proposition 3.1. Let $f: \mathbb{R} \rightarrow \mathbb{R}$ be differentiable function and $\left|f^{\prime}\right|$ be integrable on $[a, b]$ and s-convex function in the third sense on $\mathbb{R}$. Suppose that $P$ is a partition of $[a, b]$. Then

$$
|E(f, P)| \leq \frac{3 s}{2\left(s^{2}+1\right)} \sum_{k=0}^{n-1}\left(\left|x_{k}\right|+\left|x_{k+1}\right|\right)^{2}\left(\left|f^{\prime}\left(x_{k}\right)\right|+\left|f^{\prime}\left(x_{k+1}\right)\right|\right)
$$

Proof. Applying Theorem 2.2 on $\left[x_{k}, x_{k+1}\right]$, we have

$$
\left|\frac{f\left(x_{k}\right)+f\left(x_{k+1}\right)}{2}-\frac{1}{x_{k+1}-x_{k}} \int_{x_{k}}^{x_{k+1}} f(x) d x\right| \leq \frac{3 s}{2\left(s^{2}+1\right)\left(x_{k+1}-x_{k}\right)}\left(\left|x_{k}\right|+\left|x_{k+1}\right|\right)^{2}\left(\left|f^{\prime}\left(x_{k}\right)\right|+\left|f^{\prime}\left(x_{k+1}\right)\right|\right) \text {. }
$$

Then using (3.1) and (3.2), we get the desired result as follows:

$$
\begin{aligned}
|E(f, P)| & =\left|\sum_{k=0}^{n-1} \frac{f\left(x_{k}\right)+f\left(x_{k+1}\right)}{2} \Delta x_{k+1}-\int_{a}^{b} f(x) d x\right| \\
& =\left|\sum_{k=0}^{n-1}\right| \frac{f\left(x_{k}\right)+f\left(x_{k+1}\right)}{2} \Delta x_{k+1}-\int_{x_{k}}^{x_{k+1}} f(x) d x \mid \\
& \leq \sum_{k=0}^{n-1}\left|\frac{f\left(x_{k}\right)+f\left(x_{k+1}\right)}{2} \Delta x_{k+1}-\int_{x_{k}}^{x_{k+1}} f(x) d x\right| \\
& =\sum_{k=0}^{n-1} \Delta x_{k+1}\left|\frac{f\left(x_{k}\right)+f\left(x_{k+1}\right)}{2}-\frac{1}{x_{k+1}-x_{k}} \int_{x_{k}}^{x_{k+1}} f(x) d x\right| .
\end{aligned}
$$

Proposition 3.2. Let $a, b$ be real numbers with $a<b$ and $f: \mathbb{R} \rightarrow \mathbb{R}$ be differentiable function such that $\left|f^{\prime}\right|^{p}$ is integrable on $[a, b]$ and s-convex function in the third sense on $\mathbb{R}$. Suppose that $P$ is a partition of $[a, b]$. Then

$$
|E(f, P)| \leq \frac{3}{2 s}\left(\frac{s^{2}}{s^{2}+1}\right)^{\frac{1}{p}} \sum_{k=0}^{n-1}\left(\left|x_{k}\right|+\left|x_{k+1}\right|\right)^{2}\left(\left|f^{\prime}\left(x_{k}\right)\right|^{p}+\left|f^{\prime}\left(x_{k+1}\right)\right|^{p}\right)^{\frac{1}{p}} .
$$

Proof. Applying Theorem 2.5 in a similar way to proof of the Proposition 3.1.

\section{CONFLicts OF INTEREST}

The author declares that there are no conflicts of interest regarding the publication of this article.

\section{Authors Contributions Statement}

The author has read and agreed to the published version of the manuscript. 


\section{REFERENCES}

[1] Adilov, G., Yesilce, I., On Generalizations of the Concept of Convexity, Hacettepe Journal of Mathematics and Statistics, 41(2012), 723-730.

[2] Adilov, G., Yesilce, I., $B^{-1}$-convex Functions, Journal of Convex Analysis, 24(2017), 505-517.

[3] Adilov, G., Yesilce, I., Some important properties of B-convex functions, Journal of Nonlinear and Convex Analysis, 19(2018), 669-680.

[4] Adilov, G. R., Kemali, S., Abstract convexity and Hermite-Hadamard type inequalities, Journal of Inequalities and Applications, 2009(2009), 943534.

[5] Alomari, M.W., Darus, M., Kirmaci, U.S., Some inequalities of Hermite-Hadamard type for s-convex functions, Acta Mathematica Scientia, 31(2011), 1643-1652.

[6] Avriel, M., r-convex Functions, Mathematical Programming, 2(1972), 309-323.

[7] Bayoumi, A., Fathy Ahmed A., p-Convex Functions in Discrete Sets, International Journal of Engineering and Applied Sciences, 4(2017), 63-66.

[8] Breckner, W.W., Stetigkeitsaussagen fü $r$ eine Klasse verallgemekterter konvexer Funktionen in topologischen linearen Raumen, Publ. Inst. Math., 23(1978), 13-20.

[9] Briec W., Horvath, C., B-convexity, Optimization, 53(2004), 103-127.

[10] Dragomir, S., Fitzpatrick, S., Hadamard's inequality for s-convex functions in the first sense and applications, Demonstratio Mathematica, 31(1998), 633-642.

[11] Dragomir, S. S., Fitzpatrick, S., The Hadamard inequalities for s-convex functions in the second sense, Demonstratio Mathematica, 32(1999), $687-696$.

[12] Eken, Z., Kemali, S., Tinaztepe, G., Adilov G., The Hermite-Hadamard Inequalities for p-Convex Functions, Hacettepe Journal of Mathematics and Statistics, 50(5)(2021), 1268-1279, https://doi.org/10.15672/hujms.775508.

[13] Gozpinar, A., Set, E., Dragomir, S S., Some generalized Hermite-Hadamard type inequalities involving fractional integral operator for functions whose second derivatives in absolute value are s-convex, Acta Mathematica Universitatis Comenianae, 88(2019), 87-100.

[14] Kemali, S., Sezer, S., Tinaztepe, G., Adilov, G., s-Convex Functions in the Third Sense, Korean J. Math., 29(3)(2021), 593-602.

[15] Kemali, S., Tinaztepe, G., Adilov, G., New Type Inequalities For B-Convex Functions Involving Hadamard Fractional Integral, Facta Universitatis-Series Mathematics And Informatics, 33(2018), 697-704.

[16] Orlicz, W., A note on modular spaces I, Bull. Acad. Polon. Soi., Ser. Math. Astronom Phys., 9(1961), 157-162.

[17] Özcan, S., Iscan, I., Some new Hermite-Hadamard type inequalities for s-convex functions and their applications, J. Inequal. Appl., 2019(2019), 201.

[18] Rockafellar, R.T., Convex Analysis, Princeton University Press, New Jersey, 1972.

[19] Rubinov, A., Abstract Convexity and Global Optimization, Nonconvex Optimization and Its Applications, Kluwer Academic Publisher, Dordrecht, The Netherlands, 2000.

[20] Sarikaya, M.Z., Kiris, M.E., Some new inequalities of Hermite-Hadamard type for s-convex functions, Miskolc Mathematical Notes, 16(2015), 491-501.

[21] Set, E., Özdemir, M., Dragomir, S., On Hadamard-type inequalities involving several kinds of convexity, Journal of Inequalities and Applications, (2010), 1-12.

[22] Set, E., Iscan, I., Kara, H.H., Hermite-Hadamard-Fejer Type Inequalities for s-Convex Function in the Second Sense via Fractional Integrals, Filomat, 30(2016), 3131-3138.

[23] Sezer, S., Eken, Z., Tinaztepe, G., Adilov, G., p-Convex Functions and Some of Their Properties, Numerical Functional Analysis and Optimization, DOI 10.1080/01630563.2021.1884876, (2021).

[24] Sezer, S., The Hermite-Hadamard Inequality for s-Convex Functions in the Third Sense, AIMS Mathematics, 6(2021), 7719-7732.

[25] Sezer, S., Hermite-Hadamard Type Inequalities for the Functions Whose Absolute Values of First Derivatives are p-Convex Function, Fundamental Journal of Mathematics and Applications, 4(2021), 88-99. 